\title{
DICER1 hotspot mutations in non-epithelial gonadal tumours
}

L Witkowski ${ }^{1,2,3}$, J Mattina ${ }^{2}$, S Schönberger ${ }^{4}$, M J Murray ${ }^{5,6}$, C S Choong ${ }^{7,8}$, D G Huntsman9 , J S Reis-Filho ${ }^{10}$, W G McCluggage ${ }^{11}$, J C Nicholson ${ }^{5}$, N Coleman ${ }^{6}$, G Calaminus ${ }^{12}$, D T Schneider ${ }^{13}$, J Arseneau ${ }^{14}$, C J R Stewart ${ }^{15}$ and W D Foulkes s, $2,3,16^{2}$

${ }^{1}$ Department of Oncology and Human Genetics, Program in Cancer Genetics, McGill University, Montreal, Quebec, Canada; ${ }^{2}$ Department of Human Genetics, Lady Davis Institute and Segal Cancer Centre, Jewish General Hospital, McGill University, Montreal, Quebec, Canada; ${ }^{3}$ Department of Human Genetics, McGill University, Montreal, Quebec, Canada; ${ }^{4}$ Department of Paediatric Haematology and Oncology, University of Bonn, University Children's Hospital, Bonn, Germany; ${ }^{5}$ Department of Paediatric Oncology and Haematology, Cambridge University Hospitals NHS Foundation Trust, Cambridge, UK; ${ }^{6}$ Department of Pathology, University of Cambridge, Cambridge, UK; ${ }^{7}$ School of Paediatrics and Child Health, University of Western Australia, Crawley, Western Australia, Australia; ${ }^{8}$ Department of Paediatric Endocrinology and Diabetes, Princess Margaret Hospital for Children, Child and Adolescent Health Service, Subiaco, Western Australia, Australia; ${ }^{9}$ Centre for Translational and Applied Genomics, The British Columbia Cancer Agency and the Genetic Pathology Evaluation Centre of the Departments of Pathology of the Vancouver General Hospital, British Columbia, Canada; ${ }^{10}$ Department of Pathology, Memorial Sloan-Kettering Cancer Centre, New York, NY, USA; ${ }^{11}$ Department of Pathology, Royal Group of Hospitals Trust, Belfast, Northern Ireland, UK; ${ }^{12}$ Department of Paediatric Haematology and Oncology, University of Münster, Münster, Germany; ${ }^{13}$ Clinic of Paediatrics, Municipal Hospital Dortmund, Dortmund, Germany; ${ }^{14}$ Department of Pathology, McGill University, Montreal, Quebec, Canada; ${ }^{15}$ King Edward Memorial Hospital, Department of Histopathology, Perth, Australia and ${ }^{16}$ Research Institute, McGill University Health Centre, Montreal, Quebec, Canada

Correction to: British Journal of Cancer (2013) 109, 2744-2750. doi:10.1038/bjc.2013.637

Owing to an error on the authors' part, the fifth named author (Dr C S Choong) was omitted from the author listing when the paper was originally published. The full, correct author listing is now shown above. 